\title{
Abortion Practices in Nepal: What does Evidence Show?
}

\author{
Bhandari TR ${ }^{1,2}$, Dangal $\mathrm{G}^{3}$ \\ ${ }^{1}$ Department of Public Health, School of Health and Allied Sciences, Pokhara University, Kaski, Nepal, ${ }^{2}$ Achutha Menon \\ Centre for Health Science Studies, Sree Chitra Tirunal Institute for Medical Sciences and Technology, Trivandrum, \\ Kerala, India, ${ }^{3}$ Department of Obstetrics and Gynaecology, Kathmandu Model Hospital, Kathmandu, Nepal
}

Received: May 15, 2014; Accepted: December 12, 2014

\begin{abstract}
Twenty-five years have passed since the global community agreed in Nairobi to address the high maternal mortality by implementing the Safe Motherhood Initiative. However, every year around 22 million women seek unsafe abortion in developing countries. Globally, the unsafe abortion accounts for $13 \%$ maternal deaths. Out of the total aborted women, around five million women were admitted to hospitals as a result of unsafe abortion. Similarly, more than three million women suffer from severe complications from unsafe abortion every year. In 2002, responding to the public voices and high attribution of unsafe abortion on maternal mortality, Nepal granted legal access to safe abortion introducing safe abortion act. Women can seek abortion up to 12 weeks of gestation for any indication. However, sex selective pregnancy termination is prohibited in Nepal. This study aimed to assess the results of various studies on abortion practices in Nepal. Literature published in PubMed, Lancet, Medline, WHO and Google Scholar web pages from 1990 to 2014 were used to prepare this paper. From 2004 to 2014, more than half a million women sought safe abortion care in Nepal. Despite the considerable progress, unsafe abortion is still a major issue in Nepal as it has been estimated that it constitutes half of all abortions undertaken every year. Published literature further showed that still an unmet need of safe abortion services exists in Nepal. However, the overall awareness of legal abortion was found to be high among Nepalese women. We found negative attitude of most people towards women who sought abortion care. Similarly, a large number of unmarried women were found at risk for seeking abortion care due to socio-cultural norms, values and stigmas in Nepal.
\end{abstract}

Keywords: abortion practices; legalization of abortion; medical abortion; surgical abortion; unsafe abortion.

\section{INTRODUCTION}

Globally, everyday approximately 800 women die from pregnancy related causes. In 2013, nearly 289,000 women died during and following pregnancy. Almost $99 \%$ deaths occurred in developing countries. ${ }^{1}$ Achieving millennium development goal five still remains a challenge in most developing countries although maternal mortality reduction has been identified as a priority agenda. ${ }^{2}$

If a woman with an unwanted pregnancy does not have access to safe abortion care, she is at a high risk of undergoing an unsafe abortion. Every year around 22 million women seek unsafe abortion and this occurs mostly in developing countries. Unsafe abortion accounts for $13 \%$ maternal deaths. Around 5 million women admit to hospitals as a result of

\section{CORRESPONDENCE}

Tulsi Ram Bhandari

Achutha Menon Centre for Health Science Studies,

Sree Chitra Tirunal Institute for Medical Sciences and

Technology, Trivandrum, Kerala, India

Email: tulsib2004@gmail.com

Phone: +977-9851178182 unsafe abortion and more than three million women who have complications following unsafe abortion do not receive skilled care each year. ${ }^{3-5}$

Despite various efforts and liberalization of abortion laws, unsafe abortion remains a major public health concern in developing countries. Access to safe abortion remains elusive for many women because of the urban centered health facilities, poor awareness, costs, cultural issues, and availability of skilled human resources. ${ }^{6,7}$

In 2002, responding to public health, human rights imperatives and the high attribution of unsafe abortion on maternal mortality, Nepal's legal code (Muluki Ain 1854) 11 ${ }^{\text {th }}$ Amendment Bill was adopted by the parliament and granted women legal access to safe abortion. ${ }^{89}$ Women can seek safe abortion up to 12 weeks of gestation for any indication upon request, up to 18 weeks of gestation in case of rape or incest and at any time during pregnancy in case of mental/ physical illness or if the life of the pregnant woman is at risk as approved by a medical practitioner and at any time during pregnancy if the fetus is deformed and incompatible with life. Additional considerations 
include- only the certified providers are eligible to provide induced abortion services. The pregnant woman alone has the right to choose to continue or discontinue pregnancy, however in the case of minors ( $<16$ years of age) or mental incompetence; a legal guardian must give consent. Moreover, sex selective pregnancy termination is extremely prohibited in Nepal. ${ }^{8-12}$

Since 2002, in order to regulate abortion laws, the Government of Nepal has initiated various efforts. In 2003, Ipas for the first time conducted training for trainers on abortion care and in the same year government approved the Safe Abortion Procedural Order for establishing safe abortion care. Safe Abortion Advisory Committee allowed the commencement of safe abortion services in approved health facilities. In 2004, legal abortion services started for the first in some selected government hospitals of Kathmandu, Nepal. In the same year, manual vacuum aspiration training course was also started for service providers. The Abortion Task Force was dissolved and a technical committee for planning and implementing the comprehensive abortion care services was formed throughout the country. In 2007 , government introduced second-trimester abortion services. Similarly, midlevel provider training was started and medical abortion scale of strategy was approved in 2007 and 2008 respectively. ${ }^{8,9,13}$

Eight hundred eight-one physicians and 371 staff nurses were trained for safe abortion care and 255 auxiliary nurse midwives received midlevel abortion training from 2002 to 2011. Similarly, 532 safe abortion care health facilities including private sectors were registered and established covering all 75 districts. At the end of 2011 throughout the country, 497,804 women sought the safe abortion. ${ }^{8,9,13}$ Despite such considerable progress, unsafe abortions are still a major issue in Nepal as it has been estimated that they constitute only half of all the abortions undertaken every year. In Nepal, estimated 97,400 illegal abortions occurred in 2008 which was likely equal to those done by unregistered providers. ${ }^{9,14}$ This indicates the unmet need of safe abortion services in Nepal. This paper examines the published research findings on abortion care practices in Nepal from 1990 to 2014 .

\section{METHODS}

For assessing the scientific publication on abortion practices in Nepal, we searched and analyzed all available scientific writings published from 1990 to 2014. We decided to start from 1990 considering that Nepal's first National Health Policy was launched in 1991. We used the various key words such as "Nepal" and "abortion/medical abortion/unsafe abortion/ pregnancy termination/access of safe abortion/ induced abortion." We developed search strategy based on search terms and used filter to confine the publication period.

The main sources for our literature searching are PubMed, ScienceDirect, Google Scholar, Cochrane Library, World Health Organization and Ministry of Health and Population of Nepal homepages. First, we collected available literature and screened them for unrelated as well as duplicate items. Second, we classified all literature into three groups, i.e. original articles, review articles and other publications. We found 58 relevant publications, out of which we located 38 original articles, six review articles and 14 other publications such as comments, talking points, editorials, view points, working papers, conference papers, unpublished thesis, reports and case reports. We selected 38 peer-reviewed original articles to assess the abortion practices in Nepal, of which five articles were based on community based studies and the remaining articles were based on the health facilities based studies (Figure 1).

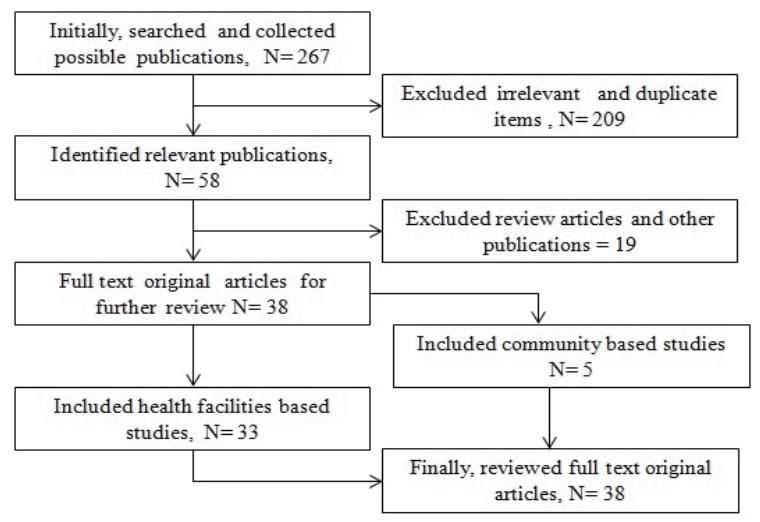

Figure 1: Flow diagram of literature search and management

\section{RESULTS}

We analyzed 38 articles considering objectives, designs, methods, study sites, sample size and major findings of the studies which are presented below (Table 1 and 2). 


\begin{tabular}{|c|c|c|c|c|c|}
\hline Studies & Objectives & Designs & Study sites & Sample size & Key findings \\
\hline $\begin{array}{l}\text { Tamang } \\
\text { and } \\
\text { Tamang } \\
2005^{15}\end{array}$ & $\begin{array}{l}\text { - Gauge current } \\
\text { awareness of the } \\
\text { availability of } \\
\text { medical abortion } \\
\text { drugs in Nepal, } \\
\text { - Explore feeling of } \\
\text { health professionals } \\
\text { about the use of } \\
\text { Medical Abortion } \\
\text { (MA) to expand } \\
\text { access to safe } \\
\text { abortion in the } \\
\text { country. }\end{array}$ & $\begin{array}{l}\text { Community } \\
\text { based } \\
\text { cross- } \\
\text { sectional } \\
\text { survey }\end{array}$ & $\begin{array}{l}\text { Across the } \\
\text { country: urban } \\
\text { and peri-urban } \\
\text { areas of Terai, } \\
\text { inner Terai and } \\
\text { Kathamndu } \\
\text { valley }\end{array}$ & $\begin{array}{l}\text { Private } \\
\text { obstetrician- } \\
\text { gynaecologists } \\
49, \text { general } \\
\text { practitioners } \\
55, \text { paramedics } \\
168, \\
\text { ayurvedic and } \\
\text { homeopathic } \\
\text { practitioners } \\
62 \text { and } \\
\text { chemists } 177\end{array}$ & $\begin{array}{l}\text { - Allopathic and } \\
\text { indigenous medicines } \\
\text { available, knowledge } \\
\text { of the availability } \\
\text { of mifepristone } \\
\text { misoprostrol was low. } \\
\text { - Many were interested in } \\
\text { MA themselves. }\end{array}$ \\
\hline $\begin{array}{l}\text { Puri } \\
\text { et al } \\
2007^{16}\end{array}$ & $\begin{array}{l}\text { Explore factors } \\
\text { associated with } \\
\text { abortion decisions } \\
\text { among young couples } \\
\text { in the context of } \\
\text { recently legalized } \\
\text { abortion in Nepal. }\end{array}$ & $\begin{array}{l}\text { Community } \\
\text { based } \\
\text { cross- } \\
\text { sectional } \\
\text { survey and } \\
\text { qualitative } \\
\text { study }\end{array}$ & $\begin{array}{l}\text { Five districts } \\
\text { of Nepal: Ilam, } \\
\text { Morang, } \\
\text { Chitwan, Kaski, } \\
\text { and Lalitpur }\end{array}$ & $\begin{array}{l}\text { For survey } \\
\text { Women- } 997 \\
\text { Men-499 } \\
\text { For qualitative } \\
\text { study } \\
\text { Women -19 } \\
\text { Men- } 11\end{array}$ & $\begin{array}{l}\text { - Half had experienced } \\
\text { an unwanted pregnancy } \\
\text { and some attempted } \\
\text { abortion, however only } \\
\text { few succeeded. } \\
\text { - In decision-making } \\
\text { husband and providers } \\
\text { played a major role. }\end{array}$ \\
\hline $\begin{array}{l}\text { Thapa } \\
\text { et al } \\
2009^{17}\end{array}$ & $\begin{array}{l}\text { Reveal the } \\
\text { understanding of } \\
\text { abortion in Nepal } \\
\text { among marginalized } \\
\text { and underserved } \\
\text { community. }\end{array}$ & $\begin{array}{l}\text { Community } \\
\text { based } \\
\text { qualitative }\end{array}$ & $\begin{array}{l}\text { Throughout } \\
\text { country } \\
\text { (selected six } \\
\text { districts and } \\
\text { NGO/INGOs) }\end{array}$ & $\begin{array}{l}\text { FGD- } 13 \\
\text { KII- } 22\end{array}$ & $\begin{array}{l}\text { - Despite misconceptions, } \\
\text { many consulted } \\
\text { providers for safe } \\
\text { abortion. } \\
\text { - Legalization of abortion } \\
\text { was not enough to } \\
\text { address the unmet need. }\end{array}$ \\
\hline $\begin{array}{l}\text { Thapa } \\
\text { and } \\
\text { Sharma } \\
2012^{18}\end{array}$ & $\begin{array}{l}\text { Assesses the } \\
\text { effectiveness of } \\
\text { programmatic } \\
\text { interventions in the } \\
\text { early years of the } \\
\text { country's abortion } \\
\text { program. }\end{array}$ & $\begin{array}{l}\text { Cross- } \\
\text { sectional } \\
\text { survey }\end{array}$ & $\begin{array}{l}\text { DHS data } 2006 \\
\text { which covered } \\
\text { whole country }\end{array}$ & $\begin{array}{l}\text { - } 9926(15- \\
44 \text { years } \\
\text { old married } \\
\text { women) }\end{array}$ & $\begin{array}{l}\text { - } 32 \% \text { were aware on safe } \\
\text { abortion and } 56 \% \text { knew } \\
\text { where to go for safe } \\
\text { abortion service (SAS). } \\
\text { - There was variation } \\
\text { by ecological regions, } \\
\text { education, age and } \\
\text { number of living } \\
\text { children. }\end{array}$ \\
\hline $\begin{array}{l}\text { Hald } \\
\text { and } \\
\text { Sonder- } \\
\text { gaard } \\
2014^{9}\end{array}$ & $\begin{array}{l}\text { Explore a local } \\
\text { community's } \\
\text { perception of } \\
\text { the situation for } \\
\text { unmarried Nepalese } \\
\text { women wanting to } \\
\text { practice their legal } \\
\text { right to abortion }\end{array}$ & $\begin{array}{l}\text { Cross- } \\
\text { sectional } \\
\text { survey / } \\
\text { qualitative } \\
\text { and } \\
\text { quantitative }\end{array}$ & $\begin{array}{l}\text { Makwanpur } \\
\text { District, Nepal. }\end{array}$ & $\begin{array}{l}\text { - For survey } \\
55 \\
\text { - For in-depth } \\
\text { interview } 16\end{array}$ & $\begin{array}{l}\text { - Overall awareness } \\
\text { of legal abortion was } \\
\text { found high. } \\
\text { - People's attitude was } \\
\text { negative to women who } \\
\text { sought abortion. } \\
\text { - Unmarried women were } \\
\text { found at higher risk to } \\
\text { seek abortion due to } \\
\text { socio-cultural norms, } \\
\text { values and stigmas. }\end{array}$ \\
\hline
\end{tabular}




\begin{tabular}{|c|c|c|c|c|c|}
\hline Studies & Objectives & Designs & Study sites & Sample size & Key findings \\
\hline $\begin{array}{l}\text { Rayamajhi } \\
\text { et al } 2003^{19}\end{array}$ & $\begin{array}{l}\text { Study the reasons of } \\
\text { abortion and outcomes } \\
\text { of unsafe abortion. }\end{array}$ & $\begin{array}{l}\text { Health facility } \\
\text { based cross- } \\
\text { sectional } \\
\text { analysis }\end{array}$ & $\begin{array}{l}\text { Tertiary level } \\
\text { hospital, Dharan }\end{array}$ & $\begin{array}{l}877 \text { abortion } \\
\text { related cases }\end{array}$ & $\begin{array}{l}\text { - } 11 \% \text { of complication was } \\
\text { sepsis. } \\
\text { - } 2 / 3 \text { abortions were } \\
\text { performed by quacks. }\end{array}$ \\
\hline $\begin{array}{l}\text { Rana et al } \\
2004^{20}\end{array}$ & $\begin{array}{l}\text { Study maternal } \\
\text { mortality and morbidity } \\
\text { in induced septic } \\
\text { abortions. }\end{array}$ & $\begin{array}{l}\text { Retrospective } \\
\text { record analysis }\end{array}$ & $\begin{array}{l}\text { Purposively } \\
\text { selected a } \\
\text { teaching hospital }\end{array}$ & $\begin{array}{l}92 \text { cases of } \\
\text { induced } \\
\text { abortion from } \\
1992 \text { to } 1999\end{array}$ & $\begin{array}{l}\text { Of induced abortion } 6 \% \\
\text { faced life-threatening } \\
\text { conditions and some died. }\end{array}$ \\
\hline $\begin{array}{l}\text { Thapa et al } \\
2007^{21}\end{array}$ & $\begin{array}{l}\text { Assess whether } \\
\text { unsafe abortions are } \\
\text { getting lesser after the } \\
\text { establishment of CAC } \\
\text { unit. }\end{array}$ & $\begin{array}{l}\text { Descriptive } \\
\text { retrospective } \\
\text { records review }\end{array}$ & $\begin{array}{l}\text { A tertiary } \\
\text { level maternity } \\
\text { hospital in } \\
\text { Kathmandu }\end{array}$ & $\begin{array}{l}12,481 \text { women's } \\
\text { records were } \\
\text { reviewed who } \\
\text { attained hospital } \\
\text { for abortion care }\end{array}$ & $\begin{array}{l}\text { There was a decline in the } \\
\text { admission of serious cases } \\
\text { of induced abortion. }\end{array}$ \\
\hline $\begin{array}{l}\text { Duwadi and } \\
\text { Shrestha } \\
2007^{22}\end{array}$ & $\begin{array}{l}\text { Determine the reasons } \\
\text { that lead to choose } \\
\text { abortion, assess the } \\
\text { involvement of partner. }\end{array}$ & $\begin{array}{l}\text { Health facilities } \\
\text { based cross- } \\
\text { sectional study }\end{array}$ & $\begin{array}{l}8 \text { clinics of } \\
\text { Family Planning } \\
\text { Association of } \\
\text { Nepal }\end{array}$ & $\begin{array}{l}304 \text { women } \\
\text { from FPAN } \\
\text { clinics }\end{array}$ & $\begin{array}{l}\text { - Maternal education was a } \\
\text { strong predictor of SAS. } \\
\text { - Half accompanied by } \\
\text { husband for CAC. }\end{array}$ \\
\hline $\begin{array}{l}\text { Vaidya and } \\
\text { Giri } 2008^{23}\end{array}$ & $\begin{array}{l}\text { Assess the morbidities } \\
\text { as results of unsafe } \\
\text { abortion after } \\
\text { legalization. }\end{array}$ & $\begin{array}{l}\text { Descriptive } \\
\text { prospective } \\
\text { study }\end{array}$ & $\begin{array}{l}\text { A tertiary level } \\
\text { maternity } \\
\text { hospital in } \\
\text { Kathmandu }\end{array}$ & $\begin{array}{l}5592 \text { women } \\
\text { who sought } \\
\text { abortion related } \\
\text { care }\end{array}$ & $\begin{array}{l}\text { - Unsafe abortion was } 2 \% \text {. } \\
\text { - } 61 \% \text { were in their second } \\
\text { trimester and peritonitis } \\
(12 \%) \text { was the major } \\
\text { morbidity. }\end{array}$ \\
\hline $\begin{array}{l}\text { Karki et al } \\
2009^{24}\end{array}$ & $\begin{array}{l}\text { Assess the feasibility } \\
\text { and acceptability of a } \\
\text { simplified mifepristone- } \\
\text { misoprostol regimen. }\end{array}$ & $\begin{array}{l}\text { Cross-sectional } \\
\text { prospective } \\
\text { data collection }\end{array}$ & $\begin{array}{l}2 \text { teaching } \\
\text { hospitals and } 2 \\
\text { family planning } \\
\text { clinics }\end{array}$ & $\begin{array}{l}400 \text { women in } \\
\text { early pregnancy }\end{array}$ & $\begin{array}{l}\text { - } 91 \% \text { had successful MA. } \\
\text { - } 89 \% \text { preferred misoprostol } \\
\text { at home. }\end{array}$ \\
\hline $\begin{array}{l}\text { Chawdhary } \\
\text { et al } 2009^{25}\end{array}$ & $\begin{array}{l}\text { Compare the efficacy } \\
\text { of mifepristone and } \\
\text { misoprostol } \\
\text { with misoprostol alone } \\
\text { for MA up to } 63 \text { days }\end{array}$ & $\begin{array}{l}\text { Quasi- } \\
\text { randomized } \\
\text { controlled trial }\end{array}$ & $\begin{array}{l}\text { A public } \\
\text { teaching } \\
\text { hospital, } \\
\text { Kathmandu. }\end{array}$ & $\begin{array}{l}\text { Group A } \\
50 \text { women and } \\
\text { group B } 50 \\
\text { women }\end{array}$ & $\begin{array}{l}\text { - Fewer side effects and a } \\
\text { more complete abortion } \\
\text { rate }(94 \%) \text { was observed } \\
\text { in group A (mifepristone } \\
\text { and vaginal misoprostol). }\end{array}$ \\
\hline $\begin{array}{l}\text { Regmi and } \\
\text { Madison } \\
2010^{26}\end{array}$ & $\begin{array}{l}\text { Evaluate patient } \\
\text { satisfaction with the } \\
\text { new second-trimester } \\
\text { abortion services in } \\
\text { Nepal. }\end{array}$ & $\begin{array}{l}\text { Purposive } \\
\text { institutions } \\
\text { based study }\end{array}$ & $\begin{array}{l}\text { One public and } \\
\text { one private } \\
\text { hospital in } \\
\text { Kathmandu }\end{array}$ & $\begin{array}{l}50 \text { women who } \\
\text { sought abortion } \\
\text { care service }\end{array}$ & $\begin{array}{l}\text { - Abortion clients were } \\
\text { satisfied and well } \\
\text { counselled. } \\
\text { - There was some lack of } \\
\text { privacy and confidentiality }\end{array}$ \\
\hline $\begin{array}{l}\text { Tuladhar } \\
\text { and Risal } \\
2010^{27}\end{array}$ & $\begin{array}{l}\text { Study the level of } \\
\text { awareness about } \\
\text { legalization of } \\
\text { abortion in women } \\
\text { attending gynecology } \\
\text { OPD. }\end{array}$ & $\begin{array}{l}\text { Descriptive } \\
\text { cross-sectional } \\
\text { study }\end{array}$ & $\begin{array}{l}\text { Purposively } \\
\text { selected a } \\
\text { teaching hospital } \\
\text { in Kathmandu }\end{array}$ & $\begin{array}{l}200 \text { women } \\
\text { who sought } \\
\text { gynecology } \\
\text { services from } \\
\text { OPD. }\end{array}$ & $\begin{array}{l}\text { - } 66 \% \text { were aware of } \\
\text { legalization. } \\
\text { - Young women with higher } \\
\text { education were more } \\
\text { aware about it. }\end{array}$ \\
\hline $\begin{array}{l}\text { Shrivastava } \\
\text { et al } 2010^{28}\end{array}$ & $\begin{array}{l}\text { Find out the profile of } \\
\text { abortion clients }\end{array}$ & $\begin{array}{l}\text { A prospective } \\
\text { study }\end{array}$ & $\begin{array}{l}\text { A tertiary level } \\
\text { maternity } \\
\text { hospital }\end{array}$ & $\begin{array}{l}57 \text { clients who } \\
\text { sought second } \\
\text { trimester }\end{array}$ & $\begin{array}{l}\text { - Most common reason of } \\
\text { abortion was multiparty } \\
(61 \%) \text {. }\end{array}$ \\
\hline $\begin{array}{l}\text { Regmi et al } \\
2010^{29}\end{array}$ & $\begin{array}{l}\text { Find out the } \\
\text { contribution of unsafe } \\
\text { abortion to maternal } \\
\text { mortality/morbidity. }\end{array}$ & $\begin{array}{l}\text { Health facility } \\
\text { based study }\end{array}$ & $\begin{array}{l}\text { A tertiary level } \\
\text { hospital, Dharan } \\
\text { Nepal }\end{array}$ & $\begin{array}{l}\text { abortion } \\
70 \text { women who } \\
\text { sought unsafe } \\
\text { abortion }\end{array}$ & $\begin{array}{l}\text { - } 52 \% \text { had high grade } \\
\text { complications. } \\
\text { - } 11 \% \text { died due to unsafe } \\
\text { abortion. }\end{array}$ \\
\hline $\begin{array}{l}\text { Lamichhane } \\
\text { et al } 201130\end{array}$ & $\begin{array}{l}\text { Examine provider's } \\
\text { perspectives on sex- } \\
\text { selective abortion on } \\
\text { legal abortion in the } \\
\text { public sector. }\end{array}$ & $\begin{array}{l}\text { Qualitative } \\
\text { study: in-depth } \\
\text { interview }\end{array}$ & $\begin{array}{l}\text { Four public } \\
\text { hospitals: two } \\
\text { in Kathmandu } \\
\text { valley and two } \\
\text { outside valley. }\end{array}$ & $\begin{array}{l}35 \text { in-depth } \\
\text { interview with } \\
\text { providers and } \\
\text { administers of } \\
\text { hospitals. }\end{array}$ & $\begin{array}{l}\text { Despite ban, sex selective } \\
\text { abortion as an increasing } \\
\text { problem. } \\
\text { - Availability of abortion, } \\
\text { USG were contributors of } \\
\text { sex selective abortion. }\end{array}$ \\
\hline $\begin{array}{l}\text { Warriner et } \\
\text { al } 2011^{31}\end{array}$ & $\begin{array}{l}\text { Assess early first- } \\
\text { trimester medical } \\
\text { abortion provided by } \\
\text { midlevel providers was } \\
\text { as safe/effective. }\end{array}$ & $\begin{array}{l}\text { Multicentre } \\
\text { randomized } \\
\text { controlled } \\
\text { equivalence } \\
\text { trial }\end{array}$ & $\begin{array}{l}\text { Five rural district } \\
\text { hospitals in } \\
\text { Nepal }\end{array}$ & $\begin{array}{l}\text { Assigned to } \\
\text { doctor- } 514 \\
\text { and midlevel } \\
\text { providers- } 518\end{array}$ & $\begin{array}{l}\text { - } 1 \% \text { were failed abortion } \\
\text { in the doctor cohort and } \\
\text { none in the midlevel } \\
\text { provider cohort. }\end{array}$ \\
\hline
\end{tabular}




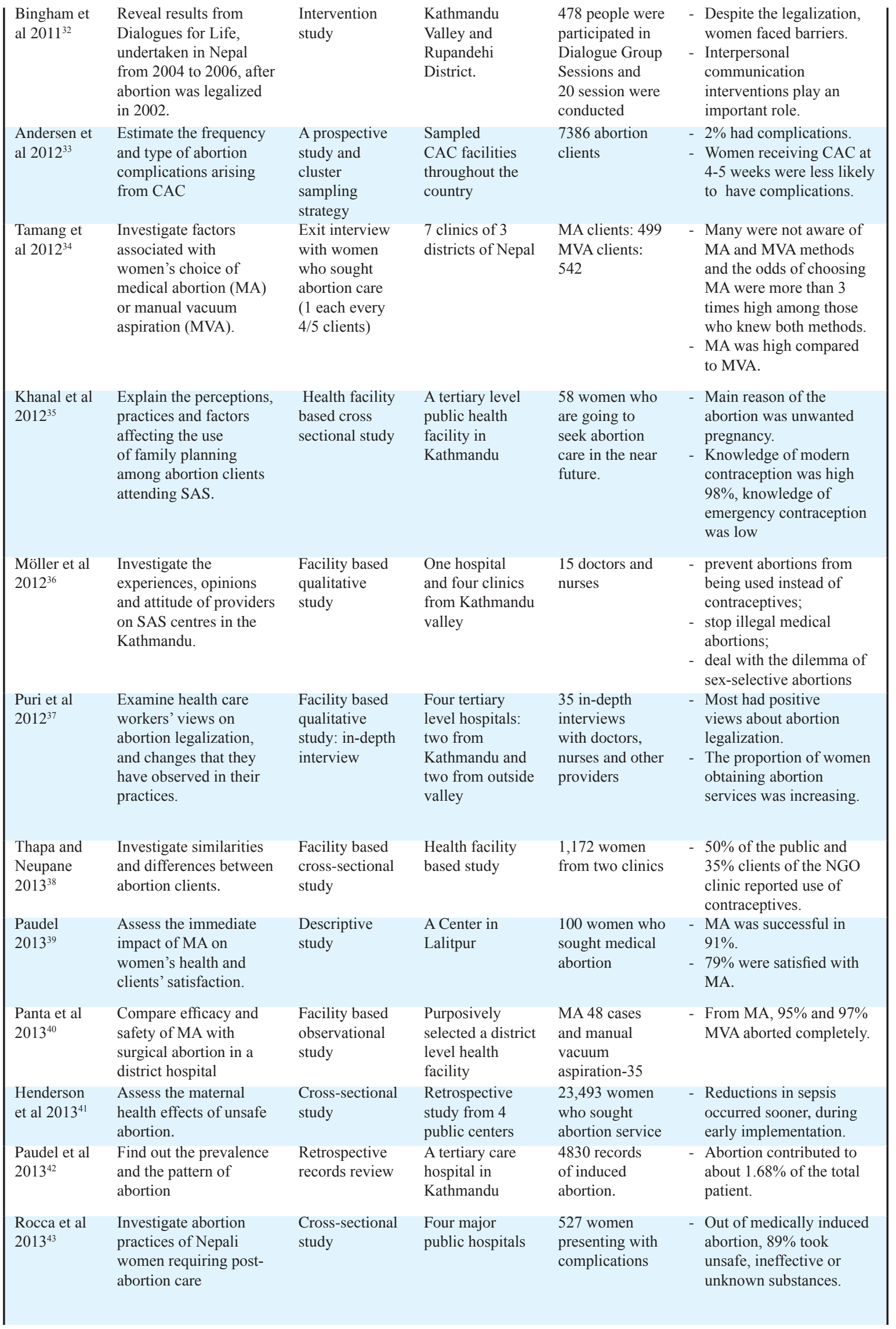




\begin{tabular}{|c|c|c|c|c|c|}
\hline $\begin{array}{l}\text { Thapa and } \\
\text { Neupane } \\
2013^{44}\end{array}$ & $\begin{array}{l}\text { Examine the incidence } \\
\text { and risk factors of repeat } \\
\text { abortion in Nepal. }\end{array}$ & $\begin{array}{l}\text { Health } \\
\text { facility based } \\
\text { prospective } \\
\text { survey }\end{array}$ & $\begin{array}{l}\text { Two approved } \\
\text { abortion clinics } \\
\text { in Kathmandu }\end{array}$ & $\begin{array}{l}1172 \text { women } \\
\text { who had surgical } \\
\text { abortions }\end{array}$ & $\begin{array}{l}\text { - One-third women had } \\
\text { repeat abortions. }\end{array}$ \\
\hline $\begin{array}{l}\text { Berin et al } \\
2014^{45}\end{array}$ & $\begin{array}{l}\text { Compare knowledge } \\
\text { and attitudes about } \\
\text { contraceptive among } \\
\text { women seeking induced } \\
\text { abortion }\end{array}$ & $\begin{array}{l}\text { Cross-sectional } \\
\text { cohort study } \\
\text { with matched } \\
\text { controls. }\end{array}$ & $\begin{array}{l}\text { Kathmandu } \\
\text { Medical College, } \\
\text { Kathmandu }\end{array}$ & $\begin{array}{l}64 \text { cases for } \\
\text { abortion service, } \\
89 \text { controls } \\
\text { (women who } \\
\text { sought medical } \\
\text { care) }\end{array}$ & $\begin{array}{l}\text { - Women with higher } \\
\text { education were less } \\
\text { likely to seek an abortion } \\
\text { than women with lower } \\
\text { education. }\end{array}$ \\
\hline $\begin{array}{l}\text { Gerdts et al } \\
2014^{46}\end{array}$ & $\begin{array}{l}\text { Assess the denial of } \\
\text { abortion in legal settings }\end{array}$ & $\begin{array}{l}\text { Cross-section } \\
\text { study }\end{array}$ & $\begin{array}{l}2 \text { abortion } \\
\text { provider clinics }\end{array}$ & $\begin{array}{l}300 \text { women } \\
\text { sought abortion }\end{array}$ & $\begin{array}{l}\text { - In Nepal, } 26 \% \text { of women } \\
\text { were denied abortions }\end{array}$ \\
\hline $\begin{array}{l}\text { Rocca et al } \\
2014^{47}\end{array}$ & $\begin{array}{l}\text { Assess contraceptive } \\
\text { information received } \\
\text { and methods chosen, } \\
\text { received, and used } \\
\text { among women having } \\
\text { abortions. }\end{array}$ & $\begin{array}{l}\text { Prospective } \\
\text { follow -up } \\
\text { study (base-line } \\
\text { and end-line } \\
\text { survey) }\end{array}$ & $\begin{array}{l}\text { Two non- } \\
\text { governmental } \\
\text { clinics and two } \\
\text { government } \\
\text { hospitals from } \\
\text { across Nepal. }\end{array}$ & $\begin{array}{l}830 \text { participated } \\
\text { from four health } \\
\text { facilities. }\end{array}$ & $\begin{array}{l}\text { - } 1 / 3 \text { did not receive } \\
\text { any information on } \\
\text { contraceptives, } 56 \% \text { left } \\
\text { facilities without any } \\
\text { method. } \\
\text { - Depo-Provera }(88 \%) \text { and } \\
\text { pills }(75 \%) \text {. }\end{array}$ \\
\hline $\begin{array}{l}\text { Puri et al } \\
2014^{48}\end{array}$ & $\begin{array}{l}\text { Determine the } \\
\text { effectiveness of } \\
\text { engaging mid-level } \\
\text { health workers to } \\
\text { provide medical } \\
\text { abortion services. }\end{array}$ & $\begin{array}{l}\text { Operation } \\
\text { research }\end{array}$ & $\begin{array}{l}\text { As study site: } \\
\text { Rupandehi } \\
\text { district and as } \\
\text { control site: } \\
\text { Kailali district }\end{array}$ & $\begin{array}{l}16 \text { health } \\
\text { facilities and } \\
126 \text { FCHVs } \\
\text { from study area } \\
\text { and } 8 \text { health } \\
\text { facilities and } 96 \\
\text { FCHVs from } \\
\text { control area }\end{array}$ & $\begin{array}{l}\text { - MA care provided by } \\
\text { nurses/ANM was reported } \\
\text { to be accessible, effective, } \\
\text { and of good quality by } \\
\text { clients who received MA. }\end{array}$ \\
\hline $\begin{array}{l}\text { Puri et al } \\
2014^{49}\end{array}$ & $\begin{array}{l}\text { Assess the effectiveness } \\
\text { of training for ANM } \\
\text { as MA providers, and } \\
\text { FCHV as referral agents } \\
\text { for expanding access } \\
\text { to MA }\end{array}$ & $\begin{array}{l}\text { Intervention } \\
\text { study } \\
\text { (comparison } \\
\text { between study } \\
\text { group and } \\
\text { control group) }\end{array}$ & $\begin{array}{l}\text { As study site: } \\
\text { Rupandehi } \\
\text { district and as } \\
\text { controlled site: } \\
\text { Kailali district }\end{array}$ & $\begin{array}{l}16 \text { health } \\
\text { facilities and } \\
126 \text { FCHVs } \\
\text { from study area } \\
\text { and } 8 \text { health } \\
\text { facilities and } 96 \\
\text { FCHVs from } \\
\text { control area }\end{array}$ & $\begin{array}{l}\text { - ANM were confident in } \\
\text { conducting MA safely and } \\
\text { effectively. } \\
\text { - FCHV were effective } \\
\text { change agents for MA. }\end{array}$ \\
\hline $\begin{array}{l}\text { Conkling et } \\
\text { al } 2014^{50}\end{array}$ & $\begin{array}{l}\text { Assess acceptability of } \\
\text { self-administration of } \\
\text { mifepristone at home. }\end{array}$ & $\begin{array}{l}\text { A prospective } \\
\text { study }\end{array}$ & $\begin{array}{l}\text { Hospital based } \\
\text { study }\end{array}$ & 200 women & $\begin{array}{l}\text { For MA, } 72 \% \text { opted to } \\
\text { take the mifepristone } \\
\text { at home from which } \\
95 \% \text { succeeded, } 94 \% \\
\text { succeeded in the clinic }\end{array}$ \\
\hline $\begin{array}{l}\text { Padmadas } \\
\text { et al } 2014^{51}\end{array}$ & $\begin{array}{l}\text { Investigate timing } \\
\text { of contraceptive } \\
\text { use and estimate } \\
\text { discontinuation rate of } \\
\text { temporary methods. }\end{array}$ & $\begin{array}{l}\text { Population- } \\
\text { based cross- } \\
\text { sectional study }\end{array}$ & $\begin{array}{l}\text { Nepal } \\
\text { Demographic } \\
\text { and Health } \\
\text { Survey data } 2011\end{array}$ & $\begin{array}{l}3190 \text { women } \\
\text { who had at least } \\
\text { one pregnancy }\end{array}$ & $\begin{array}{l}\text { - } 43 \% \text { had not initiated } \\
\text { contraceptive use in } \\
\text { following } 12 \text { months. } \\
\text { - The discontinuation rate } \\
\text { was higher in the post- } \\
\text { abortion group. }\end{array}$ \\
\hline
\end{tabular}

Most scientific studies and publications on abortion practices in Nepal were conducted and published after legalization of the abortion care services since 2002. Nearly $90 \%$ studies were conducted in the health facilities of Kathmandu valley and other big cities and followed quantitative techniques. Nearly three-fourths papers were published in the last five years. Out of the total scientific publications more than two-thirds were published in international journals. Similarly, out of the total publications in national as well as international journals, the first authors of more than two-thirds of publications were also Nepalese researchers.

\section{DISCUSSION}

This study was focused to assess the abortion practices in Nepal and relevant scientific publications before and after the legalization of abortion care services. After analysis of available literature, we concluded that safe abortion practice has been increasing steeply since legalization of abortion care services in the country. However, unsafe abortions still take place in 
various parts of the country, particularly in rural and remote areas.

More than two-third women were aware of legalization of abortion in Nepal. Studies showed

that the medical abortion has high successful rate and fewer side effects, even though both medical and surgical abortions are being practiced in Nepal. For seeking the abortion care husband and health service providers played a major role in the decision making process $^{16,25,29}$ which emphasizes the empowerment of women and their reproductive autonomy.

We noticed significant variation in abortion practices by ecological and development sub-regions, residence, education, household wealth quintile, age and number of living children. Merely legalization of abortion is not enough to address the unmet need of safe abortion and reduce the burden of unsafe abortion. ${ }^{18,42,51}$

The decision making for abortion was found to be at risk for most of the Nepalese women due to socio-cultural norms, values and stigmas; however, unmarried women were found to be at higher risk. Still, proportion of unwanted pregnancy is higher in Nepal. ${ }^{9,27,32}$ A study showed that one-third of women did not receive any information on contraceptive methods, and 56\% left facilities without any method from public health facilities. ${ }^{47}$ Many women did not use any contraceptives before termination of pregnancy and nearly one-third women had repeated abortion $^{17,22,28,35}$ which indicates that many women sought abortion as alternative of family planning. It further gives us an idea about unmet need of family planning services in Nepal. Similarly, more than half of the women did not initiate contraceptive methods after abortion. ${ }^{51}$

Nearly two-third unsafe abortions were reported in second trimester and pelvic peritonitis was the major cause of morbidities. However, there was a downward trend in the proportion of serious infection, injury and systemic complications ${ }^{19,21,23}$ which reflects the increased access of safe abortion care services in Nepal. More than two-thirds women were aware of sex-selective abortion in Nepal. Despite the strong restriction of sex selective abortion, the high sex ratio difference of the new-borne child indicates that the sex-selective abortion is being practicing consistently in Nepal. The increased access to abortion services and sex screening technology along with high value of male child in the society are contributing for sex selective abortion in Nepal. ${ }^{30}$

Despite the intensive review, we confined our search only on the free accessed literature and limited paid accessed WebPages from 1990 to 2014. So, this paper may not cover all the published literature on abortion care practices of Nepal. Out of the accessed literature, this paper primarily concentrated on the original articles either conducted in community or in health facility. The analysis focused mainly on objective, design, methods, sample size, study sites and major findings of the studies. However, this paper reveals the existing situation of abortion care practices in Nepal and scientific publications on this issue. For further assessment of the scientific literature on abortion practices of Nepal, we suggest more systematic reviews as well as meta-analyses studies.

\section{CONCLUSIONS}

After legalization of abortion care in Nepal, the utilization of safe abortion care was found to have increased steeply. Many abortion care health facilities were established and a large numbers of health care providers were trained in abortion care. Despite the various efforts, a significant number of unsafe abortion practices still exist in Nepal. Poor access to the abortion care services in remote and rural areas and socio-cultural norms, values, stigmas and so forth are the major contributing factors for unsafe abortion practices in Nepal. For addressing the unsafe abortion issues, state should ensure that all women should have access to the safe abortion services. The abortion should not be promoted instead of contraceptive methods and sex-selective abortion most be prohibited strictly.

\section{ACKNOWLEDGEMENTS}

We express sincere appreciation to all authors and publishers of these reviewed articles which are the soul of this article. Similarly, our sincere gratitude goes to Sree Chitra Tirunal Institute for Medical Sciences and Technology, Trivandrum, Kerala, India for the access of resources during reviewing. We also thank Dr. Rolina Dhital, Patan Academy of Health Science, Lalitpur, Nepal for her constructive comments on the earlier version of this article. 


\section{DISCLOSURE}

The authors report no conflicts of interest in this work.

No violation of human rights and safety.

Funding: Nil

\section{REFERENCES}

1. World Health Organization. Maternal mortality. Geneva WHO. 2014. [cited 2015 Feb 2]. Available from: http://www. who.int/mediacentre/factsheets/fs348/en/

2. Bhandari TR, Dangal G. Emergency obstetric care: strategy for reducing maternal mortality in developing countries. 2014. [cited 2014 Dec 23]. Available from: http://clacaidigital. info:8080/xmlui/handle/123456789/627

3. Zaidi S, Begum F, Tank J, Chaudhury P, Yasmin H, Dissanayake M. Achievements of the FIGO initiative for the prevention of unsafe abortion and its consequences in SouthSoutheast Asia. Int J Gynaecol Obstet Off Organ Int Fed Gynaecol Obstet. 2014;126 Suppl 1:S20-3.

4. World Health Organization. Safe abortion: technical and policy guidance for health systems. Geneva: World Health Organization; 2012.

5. Åhman E, Shah IH, World Health Organization, Special Programme of Research D, and Research Training in Human Reproduction (World Health Organization). Unsafe abortion: global and regional estimates of the incidence of unsafe abortion and associated mortality in 2008. Geneva, Switzerland: World Health Organization; 2011

6. Ngo TD, Park MH, Free C. Safety and effectiveness of termination services performed by doctors versus midlevel providers: a systematic review and analysis. Int $\mathrm{J}$ Womens Health. 2013;5:9-17.

7. Chong Y-S, Tan E-K. Midlevel health-care providers key to MDG 5. The Lancet. 2011;377:1127-8.

8. Samandari G, Wolf M, Basnett I, Hyman A, Andersen K. Implementation of legal abortion in Nepal: a model for rapid scale-up of high-quality care. Reprod Health. 2012;9:1-11.

9. Hald SC, Sondergaard DA. A gap between Law and Practice: a community's perception of unmarried women's abortion options in Nepal. Health Prospect. 2014;12:24-30.

10. Shakya G, Kishore S, Bird C, Barak J. Abortion law reform in Nepal: women's right to life and health. Reprod Health Matters. 2004;12:75-84.

11. Thapa S. Abortion law in Nepal: the road to reform. Reprod Health Matters. 2004; 12:85-94.

12. Thapa S, Sharma SK, Khatiwada N. Women's knowledge of abortion law and availability of services in Nepal. J Biosoc Sci. 2014;46:266-77.

13. Ministry of Health and Population [Nepal], New ERA, and Macro International Inc. Nepal Demographic and Health Survey 2011. Kathmandu, Nepal: Ministry of Health and Population, New ERA, and ICF International, Calverton, Maryland; 2012.

14. Sedgh G, Singh S, Henshaw SK, Bankole A. Legal abortion worldwide in 2008: levels and recent trends. Perspect Sex Reprod Health. 2011;43:188-98.

15. Tamang A, Tamang J. Availability and acceptability of medica abortion in Nepal: health care providers' perspectives. Reprod Health Matters. 2005; 13:110-9.
16. Puri M, Ingham R, Matthews Z. Factors affecting abortion decisions among young couples in Nepal. J Adolesc Health. 2007;40:535-42.

17. Thapa K, Karki Y, Bista KP. Myths and Misconceptions about abortion among marginalized underserved community. J Nepal Med Assoc. 2009;48. [cited 2015 Feb 4]. Available from: http://jnma.com.np/jnma/index.php/jnma/article/ viewFile/353/520

18. Thapa S, Sharma SK. Women's awareness of liberalization of abortion law and knowledge of place for obtaining services in Nepal. Asia Pac J Public Health. 2012;1010539512454165.

19. Rayamajhi A, Uprety J, Basnet JV. Safe abortion servicesneed of the day. [cited 2015 Mar 2]; Available from: http:// www.jnhrc.com.np/files/2003/vol1_number3_october2003/6. pdf

20. Rana A, Pradhan N, Gurung G, Singh M. Induced septic abortion: a major factor in maternal mortality and morbidity. $\mathrm{J}$ Obstet Gynaecol Res. 2004;30:3-8.

21. Thapa S, Satyal I, Malla K. Safe abortion service and post abortion care: understanding complications. Nepal J Obstet Gynaecol. 2007;2:44-9.

22. Duwadi N, Shrestha PS. Safe abortion services in Nepal: some insights. 2007 [cited 2015 Mar 2]; Available from: http://imsear.li.mahidol.ac.th/handle/123456789/46766

23. Giri A, Vaidya A. Unsafe abortion a neglected tragedy. J Nepal Health Res Counc.2009 [cited 2015 Mar 3]. Available from: http://www.jnhrc.com.np/index.php/jnhrc/article/ download/156/153

24. Karki C, Pokharel H, Kushwaha A, Manandhar D, Bracken H, Winikoff B. Acceptability and feasibility of medical abortion in Nepal. Int J Gynecol Obstet. 2009;106:39-42.

25. Chawdhary R, Rana A, Pradhan N. Mifepristone plus vaginal misoprostol vs vaginal misoprostol alone for medical abortion in gestation 63 days or less in Nepalese women: A quasi-randomized controlled trial. J Obstet Gynaecol Res. 2009;35:78-85

26. Regmi K, Madison J. Ensuring patient satisfaction with second-trimester abortion in resource-poor settings. Int $\mathrm{J}$ Gynecol Obstet. 2010;108:44-7.

27. Tuladhar H, Risal A. Level of awareness about legalization of abortion in Nepal: A study at Nepal Medical College Teaching Hospital. Nepal Med Coll J. 2010;12:76.

28. Shrivastava V, Bajracharya L, Thapa S. Surgical abortion in second trimester: initial experiences in Nepal. Kathmandu Univ Med J. 2010;8:169-72.

29. Regmi MC, Rijal P, Subedi SS, Uprety D, Budathoki B, Agrawal A. Unsafe abortion-a tragic saga of maternal suffering. J Nepal Med Assoc. 2010;49. [cited 2015 Mar 5]. Available from: http://www.jnma.com.np/jnma/index.php/ jnma/article/download/127/478

30. Lamichhane P, Harken T, Puri M, Darney PD, Blum M, Harper CC, et al. Sex-selective abortion in Nepal: a qualitative study of health workers' perspectives. Womens Health Issues. 2011;21:S37-41. 
31. Warriner IK, Wang D, Huong NM, Thapa K, Tamang A, Shah I et al. Can midlevel health-care providers administer early medical abortion as safely and effectively as doctors? A randomised controlled equivalence trial in Nepal. The Lancet 2011;377:1155-61.

32. Bingham A, Drake JK, Goodyear L, Gopinath CY, Kaufman $\mathrm{A}$, Bhattarai S. The role of interpersonal communication in preventing unsafe abortion in communities: the dialogues for life project in Nepal. J Health Commun. 2011;16:245-63.

33. Andersen K, Ganatra B, Stucke S, Basnett I, Karki YB, Thapa $\mathrm{K}$. A prospective study of complications from comprehensive abortion care services in Nepal. BMC Public Health. 2012;12:9.

34. Tamang A, Tuladhar S, Tamang J, Ganatra B, Dulal B. Factors associated with choice of medical or surgical abortion among women in Nepal. Int J Gynecol Obstet. 2012;118:S52-6.

35. Khanal V, Joshi C, Neupane D, Karkee R. Practices and perceptions on contraception acceptance among clients availing safe abortion services in Nepal. Kathmandu Univ Med J. 2012;9:179-84.

36. Moller A, Ofverstedt S, Siwe K. Proud, not yet satisfied: The experiences of abortion service providers in the Kathmandu Valley, Nepal. Sex Reprod Health. 2012;3:135-40.

37. Puri M, Lamichhane P, Harken T, Blum M, Harper CC, Darney PD et al. "Sometimes they used to whisper in our ears": health care workers' perceptions of the effects of abortion legalization in Nepal. BMC Public Health. 2012;12:297.

38. Thapa S, Neupane S. Abortion clients of a public-sector clinic and a non-governmental organization clinic in Nepal. J Health Popul Nutr. 2013;31:376-87.

39. 39. Paudel N. Immediate impact of medical abortion on women's health and clients' satisfaction with medical abortion. J Kathmandu Med Coll. 2013;1:91-5.

40. Panta OP, Bhattarai D, Parajuli N. Medical abortion versus manual vacuum aspiration in a hilly district hospital of eastern Nepal: a comparative study. Kathmandu Univ Med J. 2013;11:206-9.

41. Henderson JT, Puri M, Blum M, Harper CC, Rana A, Gurung $\mathrm{G}$ et al. Effects of abortion legalization in Nepal, 2001-2010. PloS One. 2013;8:e64775.
42. Paudel P, Paudel L, Bhochhibhoya M, Vaidhya SA, Shah N, Khatiwada D. Pattern of abortion care in a tertiary level maternity hospital in Nepal. J Nepal Med Assoc. 2013;52. [cited 2015 Feb 27]. Available from: http://jnma.com.np/ jnma/index.php/jnma/article/viewFile/2262/1628

43. Rocca CH, Puri M, Dulal B, Bajracharya L, Harper CC, Blum $\mathrm{M}$, et al. Unsafe abortion after legalisation in Nepal: a crosssectional study of women presenting to hospitals. Int J Obstet Gynaecol. 2013;120:1075-84.

44. Thapa S, Neupane S. Risk factors for repeat abortion in Nepal. Int J Gynecol Obstet. 2013;120:32-6.

45. Berin E, Sundell M, Karki C, Brynhildsen J, Hammar M. Contraceptive knowledge and attitudes among women seeking induced abortion in Kathmandu, Nepal. Int J Womens Health. 2014;6:335-41.

46. Gerdts C, DePiñeres T, Hajri S, Harries J, Hossain A, Puri $\mathrm{M}$, et al. Denial of abortion in legal settings. J Fam Plann Reprod Health Care. Published online 15 Dec 2014. [cited 2015 Feb 27 ] Available from: http://jfprhc.bmj.com/content/ early/2014/12/15/jfprhc-2014-100999.full.pdf + html.

47. Rocca CH, Puri M, Harper CC, Blum M, Dulal B, Henderson JT. Postabortion contraception a decade after legalization of abortion in Nepal. Int J Gynaecol Obstet. 2014;126:170-4

48. Puri M, Regmi S, Tamang A, Shrestha P. Road map to scalingup: translating operations research study's results into actions for expanding medical abortion services in rural health facilities in Nepal. Health Res Policy Syst. 2014;12:24.

49. Puri M, Tamang A, Shrestha P, Joshid D. The role of auxiliary nurse-midwives and community health volunteers in expanding access to medical abortion in rural Nepal.2014 [cited 2015 Mar 3]. Available from: http://www.rhm-elsevier. $\mathrm{com} / \mathrm{pb} /$ assets/raw/Health\%20Advance/journals/rhm/ RHM43S-784-Puri.pdf

50. Conkling K, Karki C, Tuladhar H, Bracken H, Winikoff B. A prospective open-label study of home use of mifepristone for medical abortion in Nepal. Int $\mathrm{J}$ Gynaecol Obstet. 2015;128:220-3.

51. Padmadas SS, Lyons-Amos M, Thapa S. Contraceptive behavior among women after abortion in Nepal. Int $\mathrm{J}$ Gynaecol Obstet. 2014;127:132-7. 